\title{
Modelling Judicial Context in Argumentation Frameworks
}

\author{
Adam WYNER ${ }^{\mathrm{a}, 1}$, Trevor BENCH-CAPON ${ }^{\mathrm{a}}$ \\ ${ }^{a}$ Department of Computer Science, University of Liverpool, Liverpool, UK.
}

\begin{abstract}
Much work using argumnentation frameworks treats arguments as entirely abstract, related by a uniform attack relation which always succeeds unless the attacker can itself be defeated. However, this does not seem adequate for legal argumentation. Some proposals have suggested regulating attack relations using preferences or values on arguments and which filter the attack relation, so that some attacks fail and so can be removed from the framework. This does not capture several important context related features of legal reasoning, such as how an audience can prefer or value an argument, yet be constrained by precedent or authority not to accept it. Nor does it explain how certain types of attack may not be allowed in a particular procedural context. For this reason, evaluation of the status of arguments within a given framework must be allowed to depend not only on the attack relations along with the preference or value of arguments, but also on the nature of the attacks and the context in which they are made. We present a means to represent these features, enabling us to account for a number of factors currently considered to be beyond the remit of formal argumentation frameworks. We give three examples of the use of approach: appealing a case, overruling a precedent, and rehearing of a case as a civil rather than criminal proceeding.
\end{abstract}

Keywords.

Argumentation, Legal reasoning, Precedent, Precedence, Procedure

\section{Introduction}

Since their introduction in [5] abstract Argumentation Frameworks (AF) have provide a fruitful tool for the analysis of the acceptability of arguments in the context of a debate comprising a set of arguments some of which conflict. In an AF, arguments are entirely abstract and related only by a uniform attack relation. This attack relation always succeeds: an argument that is attacked can be accepted only if an argument can be found to defeat its attackers. For some applications, however, such as legal argumentation which will be the focus of this paper, it is useful to allow attacks to fail. Since a court must reach a decision, it requires a rational basis for deciding, for example, between a pair of mutually attacking arguments. For this reason, AFs have been enriched to allow attacks to succeed or fail depending on properties of the arguments involved as in preference-

\footnotetext{
${ }^{1}$ Corresponding Author: Department of Computer Science, University of Liverpool, L69 3BX, UK. Tel.: +44 (0)151 795 4294; Fax: +44 (0)151 795 4235; E-mail: azwyner@liverpool.ac.uk. During the writing of this paper, the first author was supported by the Estrella Project (The European project for Standardized Transparent Representations in order to Extend Legal Accessibility (Estrella, IST-2004-027655)).
} 
based AF (PAF) of [1] or value-based AF (VAF) of [3]. In effect, the success or failure of the attack is filtered by these properties so that unsuccessful attacks may be removed, and the results of standard AFs applied. While, however, VAFs are able to accommodate reasoned choice based on legal principles or social purposes, there are other aspects of legal argumentation, in particular, the notions of precedent, precedence, and procedure as found in juridical hierarchies which are not addressed. Precedent here refers to cases which are decided by a court at one point and subsequently come under consideration by another court. Precedence refers to the hierarchical relationships between courts. Finally, procedure refers to what arguments a court finds admissible relative to some proof standard. In some contexts, while a court may be sympathetic to an argument, the court cannot accept it because that court is obligated to follow a previous decision (precedent) or a decision made by a superior court (precedence). Whether an argument can be considered also can vary: an argument which can be admitted in civil proceedings may be inadmissible in criminal proceedings, which require a higher standard of proof. The nature of the appeals process also means that different courts are able to come to different decisions on the same set of arguments. Given these observations, we can see that the evaluation of the status of arguments within a given framework must be allowed to depend not only on the attack relations, nor only on these together with the intrinsic strength of arguments relative to an audience, but also on the ways in which attacks may succeed or fail relative to the contexts in which the arguments and attacks appear. In this paper we will propose a method for accommodating these features using further extensions to AFs.

A set of cases has previously been represented as an AF in [2] and as a VAF in [4]. A means of rewriting VAFs by adding certain auxiliary arguments so that both the object level arguments and arguments expressing preferences between values was given in [7]. In this paper we will provide interpretations of these auxiliary arguments as well as constraints and processes on the construction of argument networks; these enable us to address the contextual issues relating to legal argumentation. It involves the ascription of additional properties to arguments and additional structure to the argument network. The properties are then used with respect to the structure to defend arguments against attackers which are weaker in the appropriate respect. Once the unsuccessful attacks have been removed, we can reduce the structure to an AF. Thus, while our analysis accounts for additional phenomena and adds additional machinery, it benefits from the theoretical results and algorithms which apply to AFs.

The structure of the paper is as follows. Section 2 contains a discussion of relevant aspects of the (English) legal system. In particular, we will describe the appeals process, change of use of precedent, and proof standards. Section 3 introduces the auxiliary arguments and their interpretation. Section 4 discusses how precedents are set with respect to values in an structured argument network. Section 5 presents the appeals process as a case moves upwards in the legal hierarchy. In Section 6, we show how we accommodate change in the law relative to social change. Proof standards are discussed in Section 7. We end with Section 8 and observations about opportunities for future work with our approach.

\section{Judicial Contexts}

In this section we consider the aspects of the English Legal System and juridical context which we address in this paper. 


\subsection{Example 1: Appeals Process}

The lowest level of the legal hierarchy is the Crown Court, where trials on indictment come before a judge and jury. The evidence, legal arguments, and the decision are given according to the procedures specified for the Crown Court. In particular, the Crown Court is bound by precedents decided by courts higher in the legal hierarchy. The decisions on points of law made in a Crown Court are not binding on any higher level, nor are they binding on other judges in another Crown Court, though they are persuasive. We may refer to a ratio decidendi as the legal principle on which the decision is based.

The difference between binding and persuasive precedents is important. A binding precedent is a decided case which a given court must follow in making a decision on the case before it, though this depends on the similarities between the cases. A persuasive precedent is one which is not binding, but which can be applied should it not conflict with a binding precedent and the court which applies the precedent chooses to do so. For our purposes, we simply assert the status of the precedent. We focus on binding precedents.

Cases decided in the Crown Court may be appealed to a higher level Court of Appeals. Cases can be reconsidered on matters of evidence or of law; for matters of law, there is a claim that the law has been misapplied, the rule of law which was applied is no longer desirable, or some application of the law was inappropriately missed. In effect, the ratio decidendi of the prior decision is somehow faulty.

At appeal, judges do not retry the case, but hear the evidence and arguments. The Court of Appeals can overturn a decision of a Crown Court. While the decisions of a Court of Appeals are binding on Crown Courts, the decisions of a higher court are binding on Courts of Appeals. Moreover, a Court of Appeal is bound by the decision of another Court of Appeal, with a range of exceptions (cf. Young $v$ Bristol Aeroplane Co Ltd [1944] KB 718). Typically a case in the Appeal Court is heard by three judges.

A case may be appealed from the Court of Appeal to the highest court - the House of Lords. The evidence and arguments are heard again, before five judges, called Law Lords. However, the Law Lords who judge the case are not bound by decisions made at either of the two lower courts. Following Practice Statement [1966] 3 All ER 77, the House of Lords is not even obligated to follow its own previous decisions. ${ }^{2}$

\subsection{Example 2: Change of Use of Precedent}

In general it is considered desirable for decisions made in previous cases to be applied in subsequent cases since this makes for consistency of treatment, a greater certainty as to what the law is, and stability in the system. This is the motivation for the ways in which precedents bind decisions as described above. On occasion, however, social changes may make it desirable that precedents are abandoned. This cannot be done lightly, but it is essential that it be possible if courts are to be able to adapt to changes in society at large. A discussion of one such landmark change in connection with a growing desire to represent the rights of women within marriage is given in [8]. Another example is provided by Miliangos $v$ George Frank (Textiles) Ltd [1976] AC 443, where the House of Lords overruled its own previous decision concerning Re United Railways [1961] AC 1007 and in favor of allowing damages to be awarded in a foreign currency. This was in

\footnotetext{
${ }^{2}$ The House of Lords is the highest level in the English Legal System. In the European Legal System, above it is the European Court of Justice, which we do not consider.
} 
response to a radical change in the exchange rate mechanism that had developed in the interrum. Prior to 1966, the House of Lords was bound to follow all its prior decisions under the principle of stare decisis; however, following the Practice Statement [1966] 3 All ER 77, the House of Lords granted itself the right to depart from its previous decisions where it seems right to do so.

\subsection{Example 3: Differences in Standards of proof}

In criminal proceedings a very high standard of proof, often expressed as "beyond reasonable doubt" is required. Depriving a citizen of his liberty is rightly considered a very serious matter, and a person is presumed innocent until guilt is established. This presumption is very strong: it should be maintained if there are any reasonable grounds for doubt. A civil proceedings, however, where the victim seeks compensation, uses a lower standard of proof, termed "balance of probabilities" or "preponderance of evidence". This difference means that on the basis of the same facts some arguments which were rejected as inadmissible by the criminal court will be considered and accepted by the civil court. There are a number of examples in fields such as rape and negligence, and perhaps most notoriously, the OJ Simpson murder case in the US. This is a rather different example of how the legal context can determine the outcome of a case.

\section{Representing Legal Context}

We take as our starting point an analysis of a body of case law which identifies the arguments presented in the cases analysed and the attack relation between them. An analysis of a set of cases pertaining to wild animals as a Dungian AF can be found in [2]. This framework was turned into a VAF in [4] by associating each of the arguments in the AF with the values that would be promoted by accepting them. We now need to adapt this framework by accommodating precedent, precedence, and procedure. We will do so by considering two fragments of the framework of [4].

First consider two arguments presented in the case Pierson v Post. One argument, $P P 1$, was that possession of a wild animal required bodily seizure, advanced for the sake of clarity. The other, PP2, was that hot pursuit should be accounted as possession, so as to encourage hunting for the sake of economic prosperity. Thus we have a pair of mutually conflicting arguments, each based on a different value.

While the representation of [4] records this as the crucial issue on which the decision turns, it does not record what the court decided: rather the VAF gives two sets of acceptable arguments, corresponding to the majority and minority opinions in Pierson $v$ Post. In fact, the court decided for $P P 1$, following the majority opinion. This decision needs to be recorded, which we do by adding another argument that attacks $P P 2$ and has the interpretation $P P 1$ defeated $P P 2$.

Next consider two arguments presented in Young $v$ Hitchens. YHI was that the court should find for the defendant because he was in competition with the plaintiff, while $\mathrm{YH} 2$ argued that the competition was unfair. Both of these arguments were based on the value of promoting economic prosperity. Again the response of the court is not recorded in [4]. In fact the court ruled $\mathrm{YH} 2$ inadmissible, as it was held that deciding what constituted unfair competition was beyond the remit of the court. Thus the argument that YH2 defeats 
$Y H 1$ is defeated by an argument to the effect that $Y H 2$ is inadmissible. This argument, is itself attacked by $Y H 2$.

Putting these two observations together we can see the attacks of the framework in [4] as effectively comprising a chain of four arguments. The situation is illustrated in Figure 1.

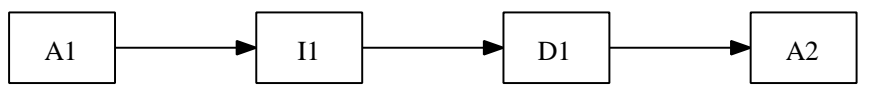

Figure 1. An Argument Chain

The arguments taken from the original analysis are indicated with A1 and A2, which we call the A-arguments or the ordinary arguments. Whereas in [4] the A-arguments attacked one another, on our new view, the attack relations between such arguments are instead mediated by intermediary arguments, relating to the possible inadmissibility of A-arguments and an assertion about which A-argument defeats the other A-argument. We have then three distinct sorts of arguments, which we refer to as A-arguments, Iarguments, and D-arguments: the attacking argument is an A-argument; an argument that this argument is inadmissible is an I-argument; an argument that the attacking argument defeats the attacked argument is a $D$-argument; and finally the attacked argument is again an A-argument. We refer to the I and D arguments as the auxiliary arguments since they are intended to facilitate reasoning about the ordinary arguments. These arguments can only attack in subsorts of the attack relation: A-arguments attack I-arguments, the A-to-I attack; I-arguments attack $D$-arguments, the I-to-D attack; and $D$-arguments attack $A$ arguments (and assuming no argument attacks itself), the D-to-A attack. Such a chain we call an A-chain, where A-arguments appear at the head (A1) and tail (A2). We defer discussion of the success of these attacks until additional machinery is introduced.

Assuming the semantics of [5], the preferred extension is $\{\mathrm{A} 1, \mathrm{D} 1\}$ : A1 attacks I1, where I1 would only hold were A1 not to be admissible; consequently, the attacker of D1 is defeated, and D1 holds; D1 attacks A2.

We enrich the A-chain by labelling the A and D arguments. Following [3], we assume that A-arguments are labelled with a value. We also want to record which of the arguments of the form $A$ defeats $B$, the $\mathrm{D}$-arguments, have in fact been endorsed by some assembly (a more general term than court) and the level of assembly which endorsed them. For example, in Pierson v Post, PP2 was endorsed by the lower court, but the decision was overturned by the appeal court which endorsed $P P 1$. Thus we would want to label the D-argument which represents $P P 1$ defeats $P P 2$ with Court of Appeal, and the D-argument which represents $P P 2$ defeats $P P 1$ with Crown Court. The values of Aarguments and the level of assembly of the D-arguments are intrinsic to the arguments. In some sense, to which we return below, the $\mathrm{D}$ arguments indirectly record the value rankings of the level of assembly which is used in determining successful attacks. In general, we label the D-arguments with the assembly in which the decision was made. This shall allow us to represent precedent. Let us suppose the labels in Figure 2. Here Unadjudicated represents that the attack of A1 on A2 has not been brought before any assembly.

Let us then assume there is a disagreement about which A-arguments win; this will arise where there are contrary claims by different parties, one party claiming that A1 


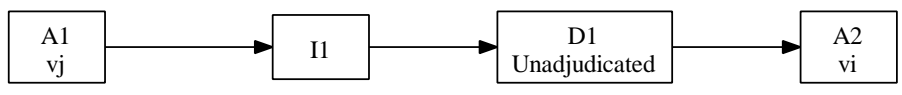

Figure 2. An Argument Chain

should defeat A2 and another that A2 should defeat A1. To represent this, we need, then, two A-argument chains. Assuming that our disagreement has not yet been brought before an assembly for adjudication, both D-arguments are labelled with Unadjudicated. We then have Figure 3, where we have two A-chains, the heads and tails attacking one another. The A-chain constituted of $\{\mathrm{A} 1, \mathrm{I} 1, \mathrm{D} 1, \mathrm{~A} 2\}$ is called the inverse of $\{\mathrm{A} 2, \mathrm{I} 2, \mathrm{D} 2, \mathrm{~A} 1\}$ and vice versa. This structure yields two preferred extensions $\{A 1, D 1\}$ and $\{A 2, D 2\}$. We are now ready to try the case and to resolve this dilemma.

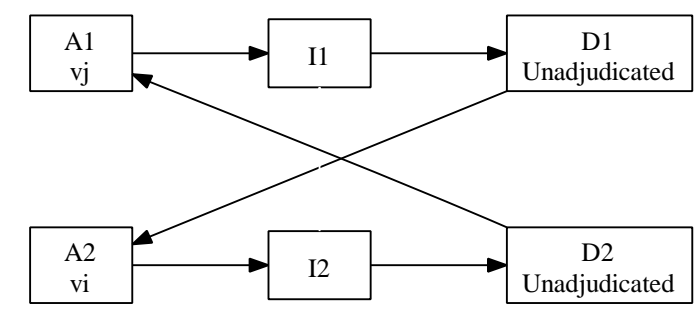

Figure 3. Mutually Attacking Arguments with Values and Level of Assembly

\section{Precedent}

At this point, we want to consider how assemblies adjudicate with respect to A-chains. We first consider where a previously Unadjudicated attack is decided by an assembly, establishing a precedent. We suppose an attack network such as in Figure 2 is brought before some assembly authorized to adjudicate, meaning that the outcome is disputed and claimed to be otherwise. Recalling that the attacked argument should be able to withstand the attack of an attacker if it relates to a more highly prized value, we add value rankings. Conceptually, these amount to attacks by a value ranking on the D-argument of an A-chain, for the head defeats the tail only where the D-argument successfully attacks the tail. This represents the relative priority given to values by the adjudicator. We associate these rankings with a level of assembly, putting aside for the moment just how the rankings and level of assembly are determined. Rather than have such value rankings external to the argument network as in [3], we introduce an additional argument sort, the $V$-arguments, which are labelled with the value ranking and the level of assembly which that ranking represents; $\mathrm{V}$-arguments are also auxiliary arguments. In addition, we have two additional subsorts of attack relations where $V$-arguments attack $D$-arguments, V-to$\mathrm{D}$ attacks, and where $V$-arguments attack $V$-arguments, V-to-V attacks. V-to-V attacks are not used in this paper, but will be needed to represent additional contextual features. V-to-D attacks are constrained with V-to-D Constraint One: V-to-D attacks can only hold where the value ranking of the $\mathrm{V}$ argument is contra the values of the head and tail of 
the A-chain of which the D-argument is a part. Intuitively, the value ranking represented in the $\mathrm{V}$-argument has to represent that argument which would defeat the D-argument given the values of the arguments in the A-chain. This overtly enforces the point that the tail is at least as highly prized as the head. More specifically, the attack of a V on D succeeds where $\mathrm{D}$ is an element of an A-chain in which the head of the chain does not have a higher value than the tail of the chain relative to the value ranking of the attacking $\mathrm{V}$; otherwise, the attack fails.

At this point, let us suppose that the attack structure in Figure 2 is brought before and decided by a Crown Court which has a value ranking of $v i>v j$. We assume that V1 attacks D1 given our constraint on V-to-D attacks. Furthermore, it is useful later to assume V-to-D Constraint Two: that for a given D-argument, there can be only one Vto-D attack. As a consequence of this attack, we construct an inverse A-chain with the $D$-argument of that A-chain labelled with the level of assembly and add it to the previous structure. Thus, the level of assembly stamps its values on the disagreement. Our system has a series of steps, each structure feeding the next. The result is Figure 4. We see how this is iterated below to represent successive steps in the judicial review process.

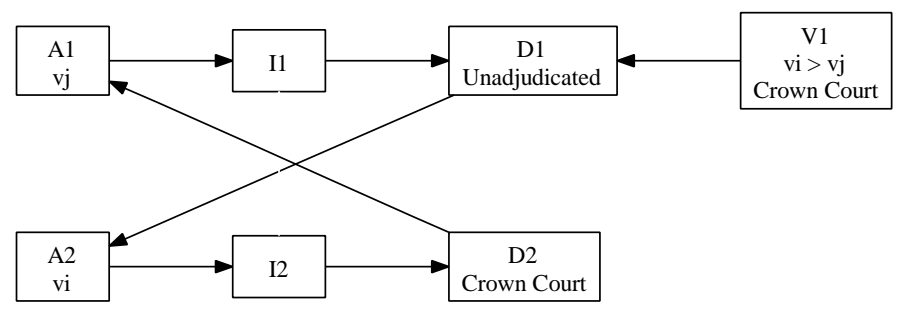

Figure 4. Structure Decided by an Assembly

While A1 attacks I1, D1 is attacked by V1, so neither I1 nor D1 hold; therefore A2 can hold. Consequently I2 does not hold (meaning that A2 is admissible), which then implies that D2 holds. D2 attacks A1, so it is out. The only preferred extension is $\{\mathrm{A} 2, \mathrm{D} 2, \mathrm{~V} 1\}$. Thus, the dilemma of choosing between A1 or A2 has been resolved according to the value preference of an assembly. Notice that the conception of successful attack varies with respect to the arguments in the attack relation and their properties: a successful V-to-D attack depends on the values of the head and tail of the A-argument chain relative to the value ranking on the $\mathrm{V}$ argument.

In this process, a first precedent has been established in the sense that a previously unadjudicated disagreement has been adjudicated, favouring one A-argument over the other.

If we consider the structure in Figure 4, we see that we have rewritten an attack between two arguments grounded on values by interposing two auxiliary arguments between them. We have also added a further auxiliary argument, the $V$ argument to attack the $\mathrm{D}$ argument. This is the structure proposed for the result of rewriting VAFs in [7] so as to overtly represent attacks on value preferences. Whereas in [7] the auxiliary arguments are abstract, here we have given an interpretation to them, which enables us to ascribe properties to them and handle issues of judicial context. 


\section{Appeals and Precedence}

We now have the essential structure we need to accommodate iterations of judicial consideration, which reflect the appeals process and precedence of one assembly over another in the judicial hierarchy. We can now take the decision represented in Figure 4 and suppose that it is appealed to the next level of the judicial hierarchy. We do this by relativising the attacks on D arguments with respect to levels of assembly. An earlier outcome (i.e. preferred extension) can be changed relative to the values or procedures of a higher court. For example, in Pierson v Post, PP2 was endorsed by the lower court, but the decision was overturned by the appeal court which endorsed $P P 1$. Thus we label the D-argument which represents PP1 defeats PP2 with Court of Appeal, and the D-argument which represents $P P 2$ defeats $P P 1$ with Crown Court. To represent the legal hierarchy which effects a change in the outcome of the disagreement relative to fixed values, we relativise the V-to-D attacks with V-to-D Constraint Three: a V-to-D attack can only appear where the level of the assembly on the V-argument and D-arguments abide by the the legal hierarchy. If the legal system requires a strict ordering, then the assembly label on a V-argument must be strictly higher than the assembly label on the attacked D-argument; if the legal hierarchy allows them to be equal, then the assembly labels on $\mathrm{V}$ and $\mathrm{D}$ can be the same.

Let us iterate the process started in Figure 4. Doing so allows us to preserve the information about the labels on the decisions as well as represent the hierarchical structure of the review process. Given our three constraints on V-to-D attacks, D2 can only be attacked by a court at the same or higher level and having a value ranking in opposition to that of the A-chain. Assuming a Court of Appeal with value ranking $v j>v i$ and our construction of an inverse A-chain, we have the three A-chains in Figure 5. The result is that the V2 attack on D2 means that A1 is reinstated. While D1, which otherwise would have defeated A2, is still eliminated by V1, we have a new attack of D3 on A2, which is associated with the Court of Appeal. The preferred set of arguments is $\{$ A1, D3, V1, V2\}. This says that while the Crown Court had one preference, the Court of Appeals had the opposite preference, and that the preference of the Court of Appeals took precedence.

It would now be possible to appeal this decision further to the House of Lords, who would be free to endorse the value preference of either of the Lower Courts and so uphold or overturn the decision. We consider this in the next section.

\section{Change in Law}

The structure in Figure 5 represents a case which has been resolved. Let us elaborate on the interpretation of the V-to-D attack. If the $\mathrm{D}$ argument represents information about the level of the court at which it was decided that the head defeats the tail, then a V argument, which is an attack on this decision, represents the values of the assembly against this decision. In effect, a V-to-D attack represents the appeals process in which the assembly attempts to impose its values with respect to the previous decision of the A-chain to bring about a different result. Our V-to-D Constraint Three captures this since only higher level assemblies can attack decisions made by lower level assemblies: Crown Courts only attack unadjudicated arguments, and V1 has imposed its values on a previously unadjudicated case by attacking D1; the Court of Appeals uses its values and ranking 


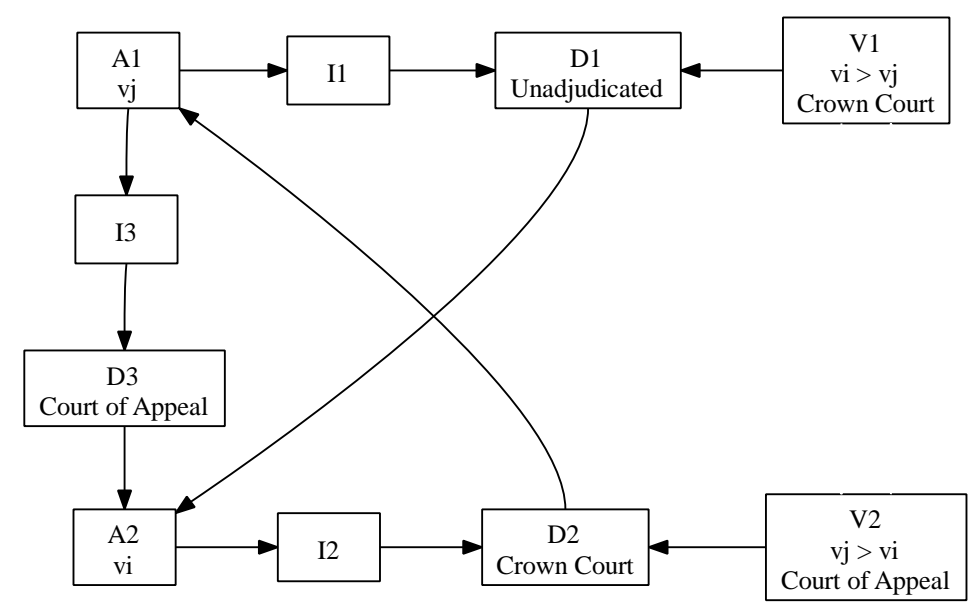

Figure 5. Defeat and Value Arguments Labelled with Courts

relative to the legal hierarchy to attack D2, in effect, overturning the decision of the Crown Court.

The decision now stands as a precedent which must be followed in subsequent similar cases. Whatever the value preference endorsed by future Crown and Appeal Courts, they are required to accept the ruling expressed in Figure 5 since they do not have the status to allow a V argument to attack D3. Suppose, however, that the inclinations of the Crown Court were the first stirrings of a social change, and in the course of time their preference became accepted throughout society. Now a case turning on A1 and A2 will be represented using the same arguments as Figure 5, but now V2 is endorsed only because precedent requires this.

At this point an appeal to the House of Lords will find that assembly subscribing to the preference $v i>v j$, which gives us a V-argument, $\mathrm{V} 3$, which is labelled for this preference and House of Lords. Following the pattern of other appeals, we introduce into Figure 5 an additional A-chain A2-I4-D4-A1, where D4 is labelled with House of Lords. This assembly is permitted to use V3 to attack D3, and so A2 is defended. As A2 defeats I4, D4 stands and defeats A1. Thus, the decision by the House of Lords reinstates and affirms the original decision of the Crown Court. This decision will stand as a precedent for subsequent cases in which a conflict between A1 and A2 is material, ensuring that the law is adapted to the changed social climate.

\section{Procedures and Proof Standards}

Precedent and Precedence made use of the D and V arguments. In order to reflect the different conditions of admissibility under different types of procedure, we make use of the I arguments. To simplify the discussion, we omit the $\mathrm{V}$-arguments and values on the A-arguments and leave aside issues related to precedent or precedence.

A-arguments which are admitted into the framework will satisfy a particular proof standard (PS) with respect to the case under consideration (see [6] and references 
therein). For our purposes, we assume four levels of PS, arranged in a hierarchy from lower to higher.

- Scintilla of Evidence (SE): supported by at least one defensible pro argument.

- Preponderance of Evidence (PE): the strongest defensible pro argument outweighs the strongest defensible con argument, if there is one.

- Dialectical Validity (DV): supported by at least one defensible pro argument and none of the con arguments are defensible.

- Beyond Reasonable Doubt (BRD): supported by at least one defensible pro argument, all of its pro arguments are defensible and none of the con arguments are defensible.

Just as we have labelled A-arguments with values, we also label the A-arguments with the PS they satisfy. These are labels which are assigned when the A-arguments appear in the argument network; we are not representing the pro and con arguments for establishing a PS of a given A-argument. As an example, in Figure 6, A1 has PS BRD while A2 has PS PE. We refer to the PS on A-arguments as the argument's evidential status. However, under different procedures, different PSs are used to determine whether an argument is admissible under that procedure: in criminal proceedings BRD is required, whereas PE is enough for civil proceedings. Thus, we are interested to represent the relationship between a given an evidential status of a particular argument and the PS in a particular procedural context. We therefore represent the procedure under which the case is being considered by labelling the I arguments in the framework with the minimum level of proof required by the procedure as determined by the assembly in which the case is being considered. Our assumption is that when an A-chain is brought before an assembly, just as the D-argument of the new inverse A-chain is labelled for the assembly which makes the decision, the I-argument of that A-chain is labelled for the PS that the assembly uses in making its decision. In Figure 6, we assume the Required Proof Standard is assigned by the procedural context; thus, when the dispute is presented in a criminal court, the required proof standard is set to BRD, while if presented in a civil court, it is PE.

We then assume that the A-to-I attack succeeds only where the evidential status of the A-argument is the same as or higher than the proof standard on the I-argument, otherwise the A-argument is inadmissible. Where the I-argument fails, the D-argument can hold (if not attacked by a V-argument). If the A-to-I attack fails, the I-argument holds, and it successfully attacks D-argument. In other words, though the head of the chain may have otherwise successfully attacked the tail, the head failed to meet the requisite proof standard, so the attack fails.

For example, in Figure 6, let us assume a criminal case, requiring the PS BRD on I1 and I2. We can start with the A-chain A1-I1-D1-A2. Note that we leave D1 to be Unadjudicated, which represents that the assembly has not made a decision since this is solely a procedural matter. As the evidential status of A1 is the same as that on I1, I1 is defeated, so D1 survives and successfully attacks A2. In turn, note that in the A-chain A2-I2-D2-A1, A2 cannot successfully attack I2, because the evidential status of A2 does not pass the PS on I2. A2 is inadmissible, and I2 survives, defeating D2. Thus, A1 is not defeated by $\mathrm{D} 2$, and the preferred extension is $\{\mathrm{A} 1\}$. Alternatively, consider where the dispute is convened under a civil procedure. The required PS changes to PE, so both I1 and I2 are labelled with PE. Again, if we start with A1, it defeats I1, so again D1 survives 
and attacks A2. However, now, A2 has a sufficient evidential status to meet the PS, so is admissible. Therefore, I2 is defeated, and D2 holds, attacking A1, which is defeated. I1 is reinstated and defeats D1, so A2 survives attack, and the preferred extension is $\{A 2\}$. By the same token, if we start with A2, the preferred extension is $\{\mathrm{A} 1\}$. We see that as the proof standards change with respect to procedural context, so changes the outcome of the argument attacks. In this latter case, the dilemma would be adjudicated according to the value preference of the assembly relative to the values on the A-arguments.

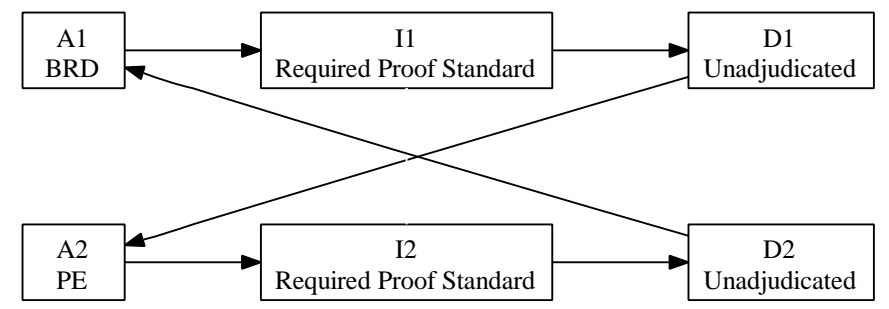

Figure 6. Standards of Proof

\section{Discussion}

In this paper we have presented an approach to handling notions of judicial context in argumentation frameworks. Our approach introduces auxiliary arguments with properties in a structured argument network, so that we are able to explicitly express decisions for argument defeat and admissibility relative to the assembly. Furthermore, by labelling the decisions and restricting attacks on D-arguments, we are able to account for precedence relations and change of law.

We have illustrated our approach with three examples: appeals and social change which show precedent and precedence, and a change in the nature of proceedings which illustrates variable admissibility. In every case, however, we have restricted ourselves to a single conflict between a pair of arguments. To move to a more complete treatment of all aspects of judicial context we need to explore the following issues.

- Work such as [4] has represented not a single pair of conflicting arguments, but the complete array of arguments that have been presented in a domain and the relations between them. These arguments may have been produced in different cases and in different contexts. We will explore how our approach can be used to represent such a body of case law, by merging particular conflicts into cases, and cases into the corpus of decisions. This has two aspects: representing the evolution of this body of law through a series of cases, and recording the current state of thinking on the topic.

- The Court of Appeals uses three judges, the House of Lords five, and the US Supreme Court nine. Multiple judges means that the individuals composing the tribunal may have different value preferences which are then composed into majority and minority blocs, the majority decision being the decision of the court. We would propose to address this by labelling the $\mathrm{V}$ arguments with the number 
of tribunal members endorsing the corresponding preference, so that the $\mathrm{V}$ argument with the greater support can succeed. Here we need V-to-V attacks. This will also permit the representation of majority and minority opinions. Minority opinions have importance as they provide arguments which may be adopted as the social climate changes.

- In our representation, failure to meet the required PS is the only source of inadmissibility. This may not always be so. For example, while an illegally conducted search may provide evidence that supports an argument to the highest standard of proof, it cannot be admitted because of the impropriety in the way the evidence was obtained. This may require us to further articulate the A-to-I attacks with auxiliary arguments, so that the I argument can be protected against the attack of the A argument. Similarly, if we wished to allow discussion as to which values should be preferred, the V-to- $\mathrm{V}$ attacks would also require auxiliary arguments.

- While our representation of judicial appeal builds a network of arguments without erasing labels as in Figure 5, the certiorari procedure allows a previous decision to be quashed and returned to an assembly for reconsideration.

- We should incorporate into the analysis burden of proof [6], which relates participants in legal contexts to the argument network.

- Auxiliary arguments, suitably labelled and in the appropriate structures and relations, could be used to represent argument schemes and critical questions [9].

These are just several of the opportunities for future work in representing judicial context. The topic is a rich one and has thus far remained beyond the reach of representation in AFs. The approach here, we believe, offers great potential to provide a well-founded representation of arguments in legal case law, and which also has promise for other areas where contextual issues are crucial in determining the status of arguments.

\section{References}

[1] L. Amgoud and C. Cayrol. On the acceptability of arguments in preference-based argumentation. In Proceedings of the 14th Annual Conference on Uncertainty in Artificial Intelligence (UAI-98), pages 1-7, San Francisco, CA, 1998. Morgan Kaufmann.

[2] T. Bench-Capon. Representation of case law as an argumentation framework. In T. Bench-Capon, A. Daskalopoulu, and R. Winkels, editors, Legal Knowledge and Information Systems, Proceedings of Jurix 2002, pages 103-112, Amsterdam, 2002. IOS Press.

[3] T. J. M. Bench-Capon. Persuasion in practical argument using value-based argumentation frameworks. J. Log. Comput., 13(3):429-448, 2003.

[4] T. J. M. Bench-Capon. Try to see it my way: Modelling persuasion in legal discourse. Artif. Intell. Law, 11(4):271-287, 2003.

[5] P. M. Dung. On the acceptability of arguments and its fundamental role in nonmonotonic reasoning, logic programming and n-person games. Artificial Intelligence, 77(2):321-358, 1995.

[6] T. Gordon, H. Prakken, and D. Walton. The carneades model of argument and burden of proof. Artificial Intelligence, 171:875-896, 2007.

[7] S. Modgil and T. Bench-Capon. Integrating object and meta-level value-based argumentation. Technical report, University of Liverpool, 2007.

[8] R. N. Moles. Definition and Rule in Legal Theory. Basil Blackwell, Oxford, 1987.

[9] D. Walton. Argumentation Schemes for Presumptive Reasoning. Erlbaum, Mahwah, N.J., 1996. 